\title{
NUEVA INSTALACIÓN DE TELECOMUNICACIONES. SANTIAGO DE COMPOSTELA (GALICIA, ESPAÑA)
}

\author{
(NEW TELECOMMUNICATIONS FACILITY. SANTIAGO DE COMPOSTELA. GALICIA, SPAIN)
}

Sir Norman Foster and Partners, Arquitectos

Fecha de recepción.: 28-VIII-95

LONDRES

\begin{tabular}{|c|c|}
\hline Arquitectos: & $\begin{array}{l}\text { - Sir Norman Foster and Partners } \\
\text { Norman Foster } \\
\text { Robin Partington } \\
\text { Etienne Borgos } \\
\text { Alistair Cook } \\
\text { Martin Cook } \\
\text { Jorge Gomendio } \\
\text { Dave Hale } \\
\text { Iwan Jones } \\
\text { Rafael Russo } \\
\text { Neil Vandersteen } \\
\text { Juan Vieira } \\
\text { Chris Windor } \\
\text { Simon Windebank } \\
\text { Richard Wotten }\end{array}$ \\
\hline Ingenieros: & - Ove Arup and Partners International \\
\hline Estructura: & $\begin{array}{l}\text { Chris Wise } \\
\text { Alistair } \\
\text { Fergus McCormick } \\
\text { Uli Mutter }\end{array}$ \\
\hline $\begin{array}{l}\text { Comunicaciones/ } \\
\text { Economía: }\end{array}$ & $\begin{array}{l}\text { - Bill Southwood } \\
\text { Christine Cox } \\
\text { Anne-Sophie Grandguillaume }\end{array}$ \\
\hline Instalaciones: & $\begin{array}{l}\text { Mohsen Zikri } \\
\text { Florence Lam } \\
\text { Ken Ma }\end{array}$ \\
\hline Investigación: & - Sarah Lovett \\
\hline Energia eólica: & - Andrew Allsop \\
\hline $\begin{array}{l}\text { Geotécnia: } \\
\text { Incendios: }\end{array}$ & $\begin{array}{l}\text { - Sergio Solera } \\
\text { - Chris Barber } \\
\text { Dominic Munro }\end{array}$ \\
\hline $\begin{array}{l}\text { Consultoria de } \\
\text { costes y presupuestos: }\end{array}$ & $\begin{array}{l}\text { Davis Langdon and Everest } \\
\text { Nick Leggett } \\
\text { Garth Hart } \\
\text { David Price } \\
\text { Simon Trafford }\end{array}$ \\
\hline Fotografía: & $\begin{array}{l}\text { - Richard Davies } \\
\text { Tom Miller }\end{array}$ \\
\hline
\end{tabular}

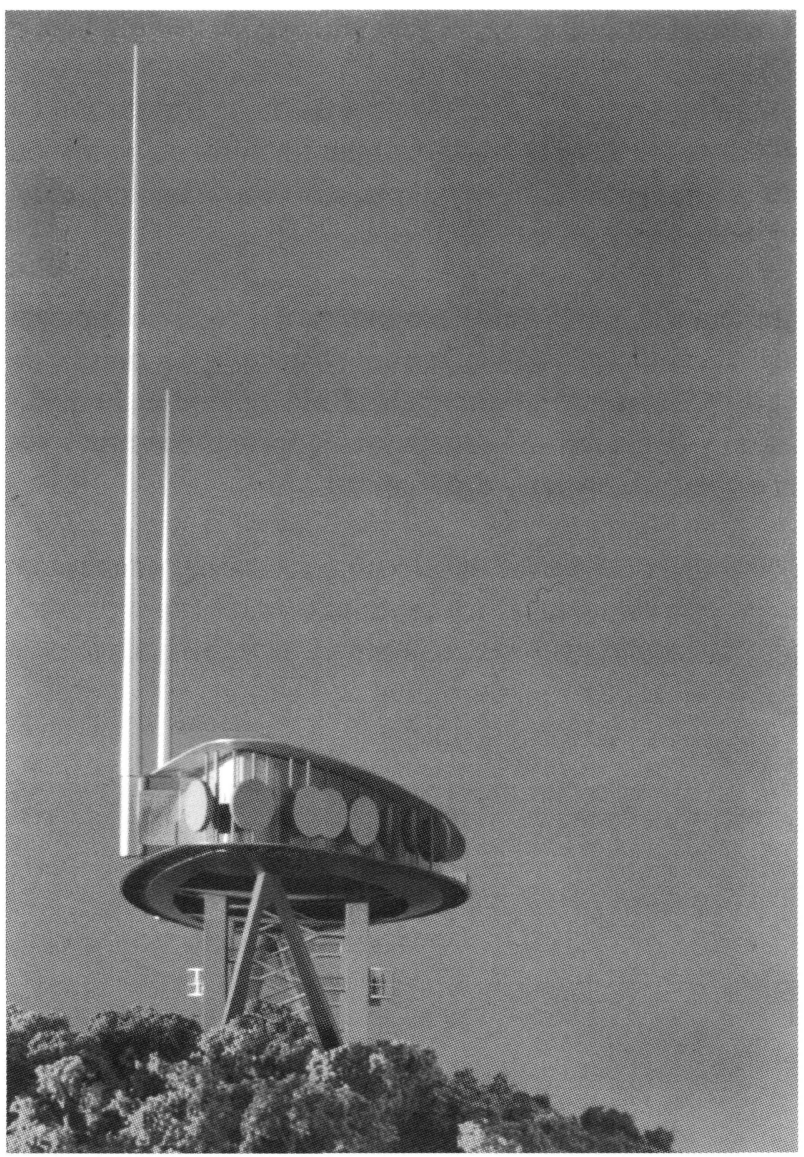

\section{RESUMEN}

La propuesta de Sir Norman Foster and Partners para el Centro de Telecomunicaciones del Monte Pedroso en Santiago de Compostela, se aleja del tipo convencional de torre de telecomunicaciones desarrollada en altura que se ha consolidado durante el último tercio del siglo XX. La arquitectura de esta clase de instalaciones, tan condicionada tecnológicamente y funcionalmente, está sometida a una contínua evolución en consonancia con el vertiginoso progreso de los sistemas de telecomunicación. El artículo expone los fundamentos del novedoso planteamiento tipológico del Centro de Telecomunicaciones de Santiago, invitando a la reflexión sobre la trascendencia que las consideraciones relativas al lugar, la función y la construcción tienen en el proceso de generación de la forma arquitectónica.

\section{SUMMARY}

The proposal of Sir Norman Foster and Partners for the Telecommunications Center of Monte Pedroso in Santiago Compostela departs from the conventional type of tall telecommunications tower, which has been consolidated in the last third of the XX century. The architecture of this kind of facilities, conditioned technologically and functionally, undergoes continuous evolution in accordance with the extremely rapid progress of the telecommunication systems. The article explains the fundamentals of the novel typological approach of the Santiago Telecommunications Center, inducing the reader to reflect on the importance that the considerations relative to the site, function and construction have in the process of generating an architectural form. 
Sir Norman Foster and Partners recibió en marzo de 1994 el encargo de diseñar una nueva instalación de telecomunicaciones para la ciudad de Santiago de Compostela en Galicia, España.

El objetivo del programa de necesidades era dotar a Santiago de una nueva instalación de telecomunicaciones que resolviera el problema generado por la rápida y antiestética proliferación de antenas de telecomunicaciones en la sierra del Monte Pedroso y que, a la vez, sirviera como centro docente y de observación para el público. El diseño resultante habría de integrarse en el Plan General del Ayuntamiento para la zona en cuestión y -a través de un programa de reforestación- devolver al Monte Pedroso su condición natural original y su carácter de uso público.

Los singulares requisitos técnicos de dicha instalación en el Monte Pedroso se tradujeron en un enfoque de diseño altamente innovador que adopta la forma de una plataforma horizontal.

Al situar a la plataforma justo encima del decisivo "umbral de línea de mira", salvándose los obstáculos del paisaje de los alrededores, se minimiza la altura general de la instalación y se posibilita la adopción de formas constructivas más convencionales y económicas.

Comparada con torres de telecomunicaciones convencionales, esta solución ofrece ventajas considerables para los diversos usuarios técnicos, proporcionando a cada uno de ellos una configuración espacial ideal para la instalación y el uso de sus equipos técnicos. Por otro lado, ofrece al público unas instalaciones que, aprovechándose del singular emplazamiento de las plataformas, disfrutan de vistas panorámicas de los alrededores, reforzando y complementando la íntima relación que el Monte mantiene con el centro histórico de la ciudad.

Las áreas técnicas de la plataforma son de altura doble, con un entresuelo en aquéllas que así lo requieran. Este entresuelo ofrece la posibilidad de ser extendido en el futuro si fuera necesario.

El espacio técnico se divide en dos zonas principales ocupadas por los usuarios mayoritarios, permitiendo que ambos dispongan de instalaciones independientes, distanciadas y aisladas de las áreas contiguas. Se podrá también acomodar a usuarios menores entre estas dos zonas.

Todos los usuarios disfrutan de acceso directo a la galería de antenas, la granja de antenas de la cubierta superior y los dos mástiles gemelos, disponiendo también de un acceso cerrado al público a sus respectivas instalaciones.

Las áreas técnicas se han configurado de manera que se propicie una relación ideal entre los transmisores y sus antenas respectivas y que se minimicen las considerables pérdidas de señal que normalmente se encuentran en las torres de telecomunicaciones convencionales.

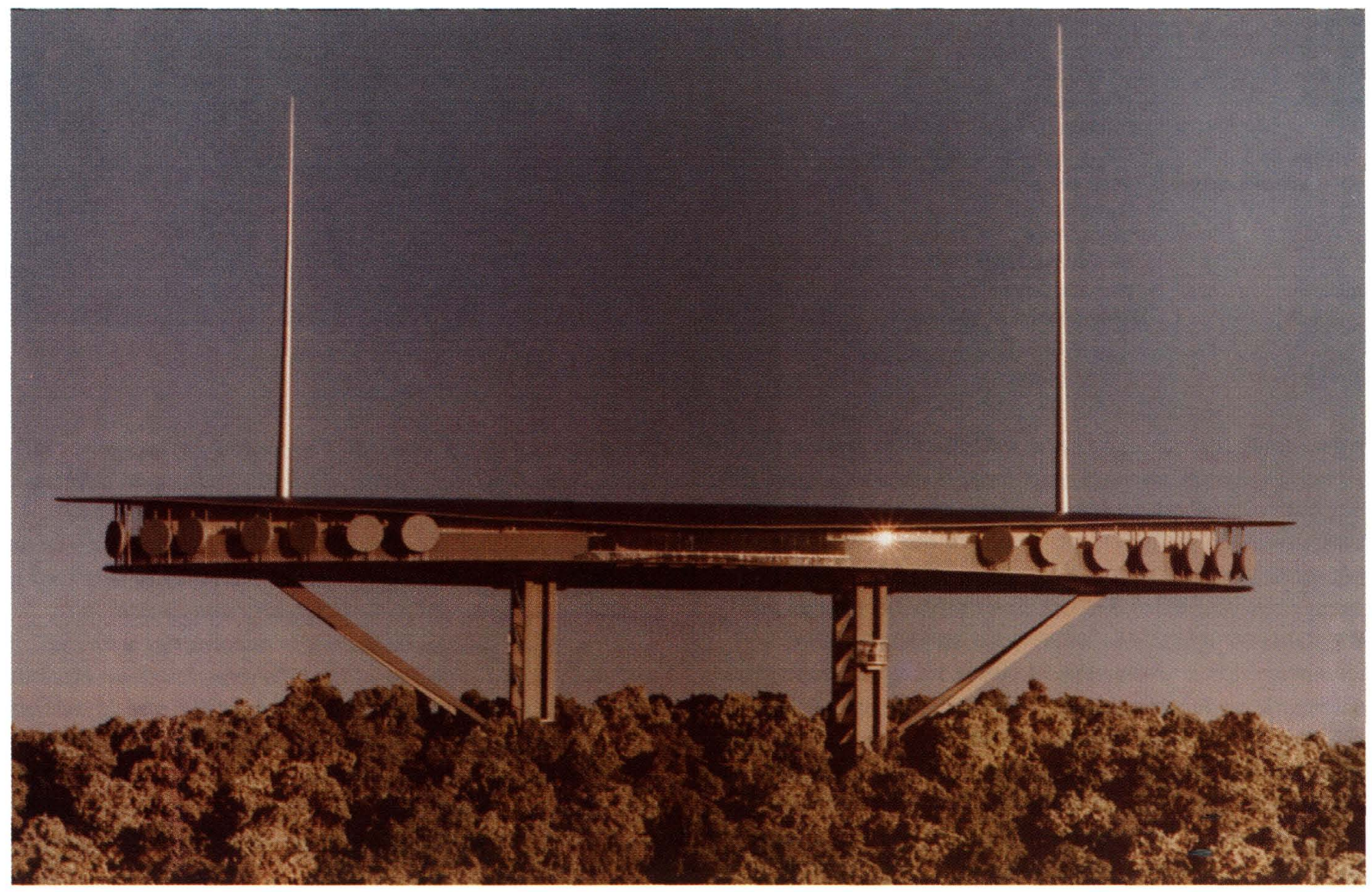




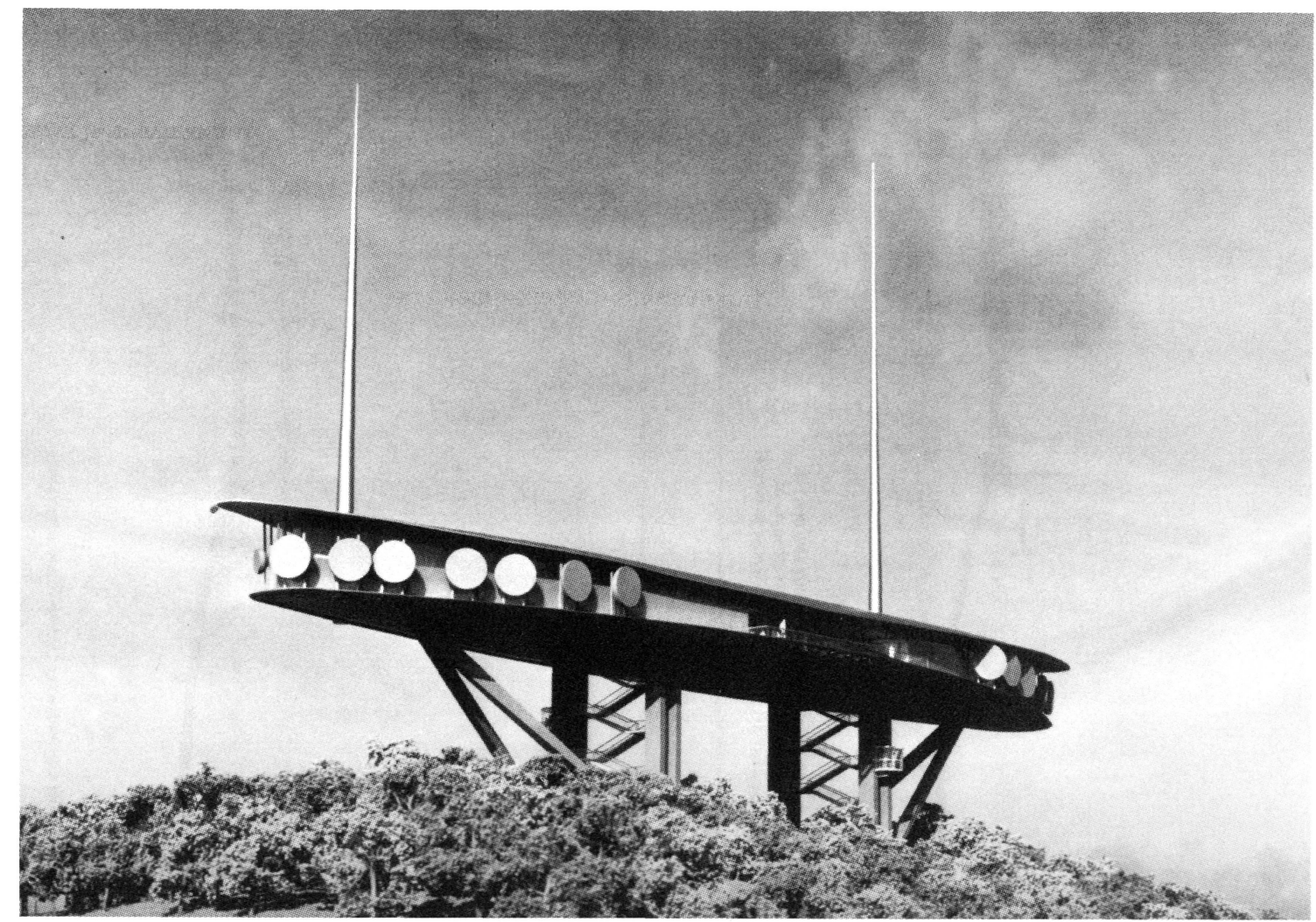

La zona de máquinas principal se ha dispuesto de manera que proporcione un servicio centralizado para cada uno de los usuarios, optimizándose así tanto el capital como los gastos de explotación. De esta manera se evita la duplicación innecesaria de máquinas y, al mismo tiempo, se mantiene un sistema de instalaciones ininterrumpido y a prueba de averías.

Ello se traduce en reducciones significativas en el consumo general de energía y en los costes de mantenimiento.

El público podrá acceder a la plataforma desde los puntos de entrada -protegidos a través de una serie de ascensores acristalados- que ofrecen una vista panorámica de los alrededores, y que ascienden, por encima del manto de las copas de árboles, a través de la parte inferior de la plataforma hasta los puntos de recepción, contiguos al vestíbulo de usos múltiples. Desde este vestíbulo, se puede subir por medio de ascensores y escaleras a una galería pública en el nivel de entresuelo, la cual envuelve tres lados del vestíbulo y conduce a una pasarela perimetral que rodea a la totalidad del edificio. Dicha pasarela ofrece $360^{\circ}$ de vistas panorámicas del paisaje de los alrededores.

Estas instalaciones públicas atienden a una variada gama de funciones -docentes, recreacionales y comerciales- e incorporan también una serie de dependencias de restauración y pequeñas unidades comerciales, así como un espacio para todo tipo de exposiciones.

Ante la continua y vertiginosa evolución a la que asistimos en el campo de las telecomunicaciones -la fibra óptica, los sistemas de cable y la radio celular-, la única constante es la necesidad de adaptarse al cambio. La plataforma que se propone para la ciudad de Santiago es diferente a las demás: se ha diseñado con el fin de proporcionar una configuración espacial ideal y a la vez ofrecer la mayor flexibilidad posible entre los usos públicos y los usos técnicos, de manera que nunca se convierta en obsoleta, garantizando así su plena utilización, tanto en el presente como en el futuro.

Al reunir a todos los usuarios en un solo edificio, se evitan los problemas acaecidos en ciudades como Los Angeles y Atenas, en las que el aumento de la demanda de telecomunicaciones ha llevado a una proliferación incontrolada de torres antiestéticas que atestan el horizonte.

Esta nueva instalación, que ha adoptado el nombre oficial de Puerto de Telecomunicaciones y Mirador del Monte Pedroso, ofrece una oportunidad única para Santiago de mantener y realzar su papel como centro de telecomunicaciones, mejorando considerablemente las instalaciones públicas de la región y erigiéndose en un símbolo para el próximo Milenio. 


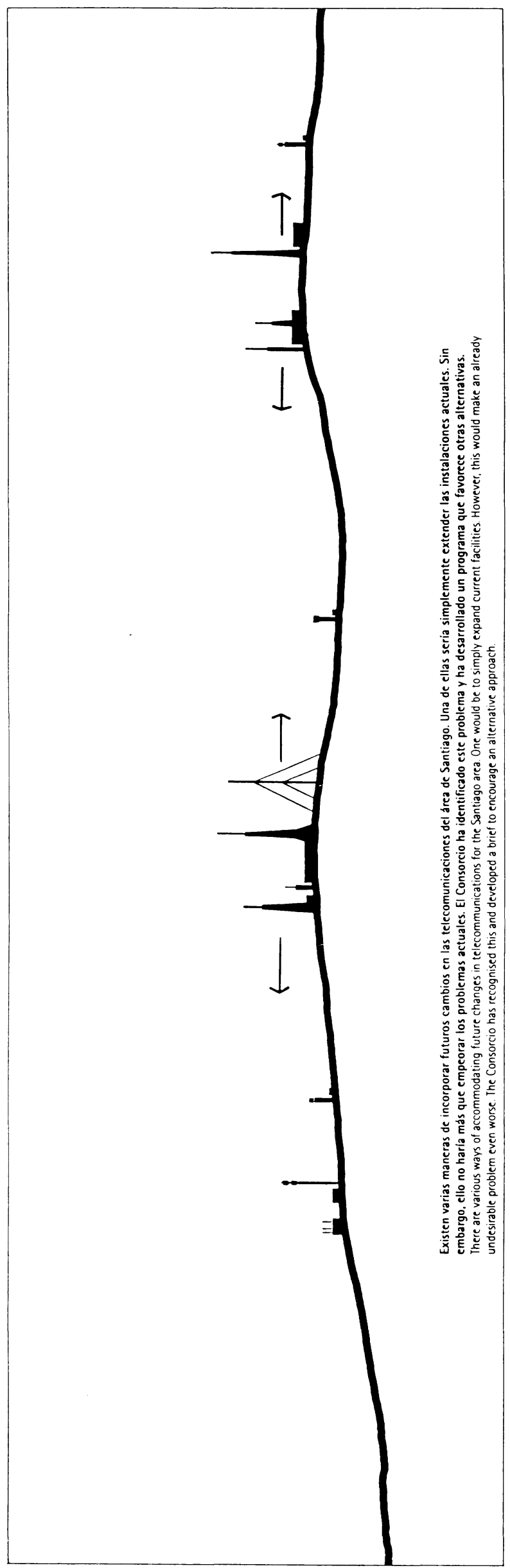

(c) Consejo Superior de Investigaciones Científicas Licencia Creative Commons 3.0 España (by-nc)

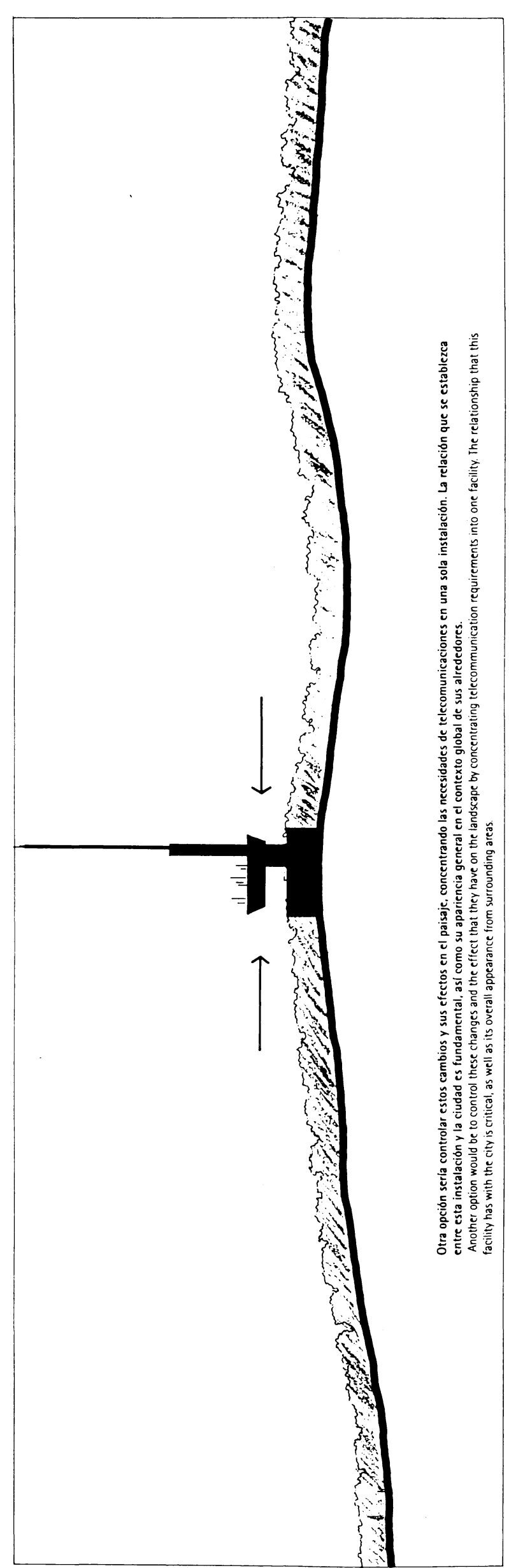

http://informesdelaconstruccion.revistas.csic.es 


$$
11
$$




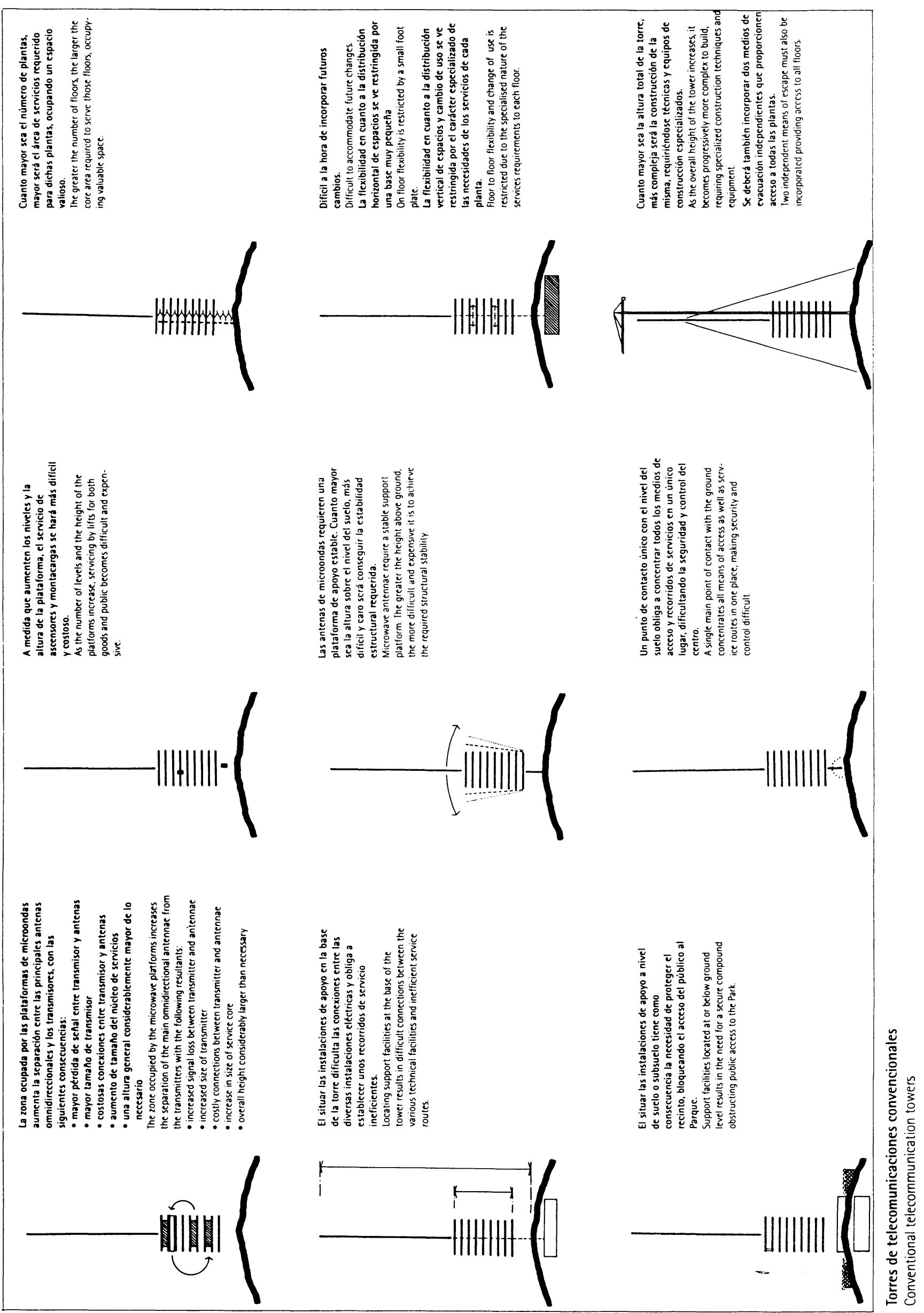




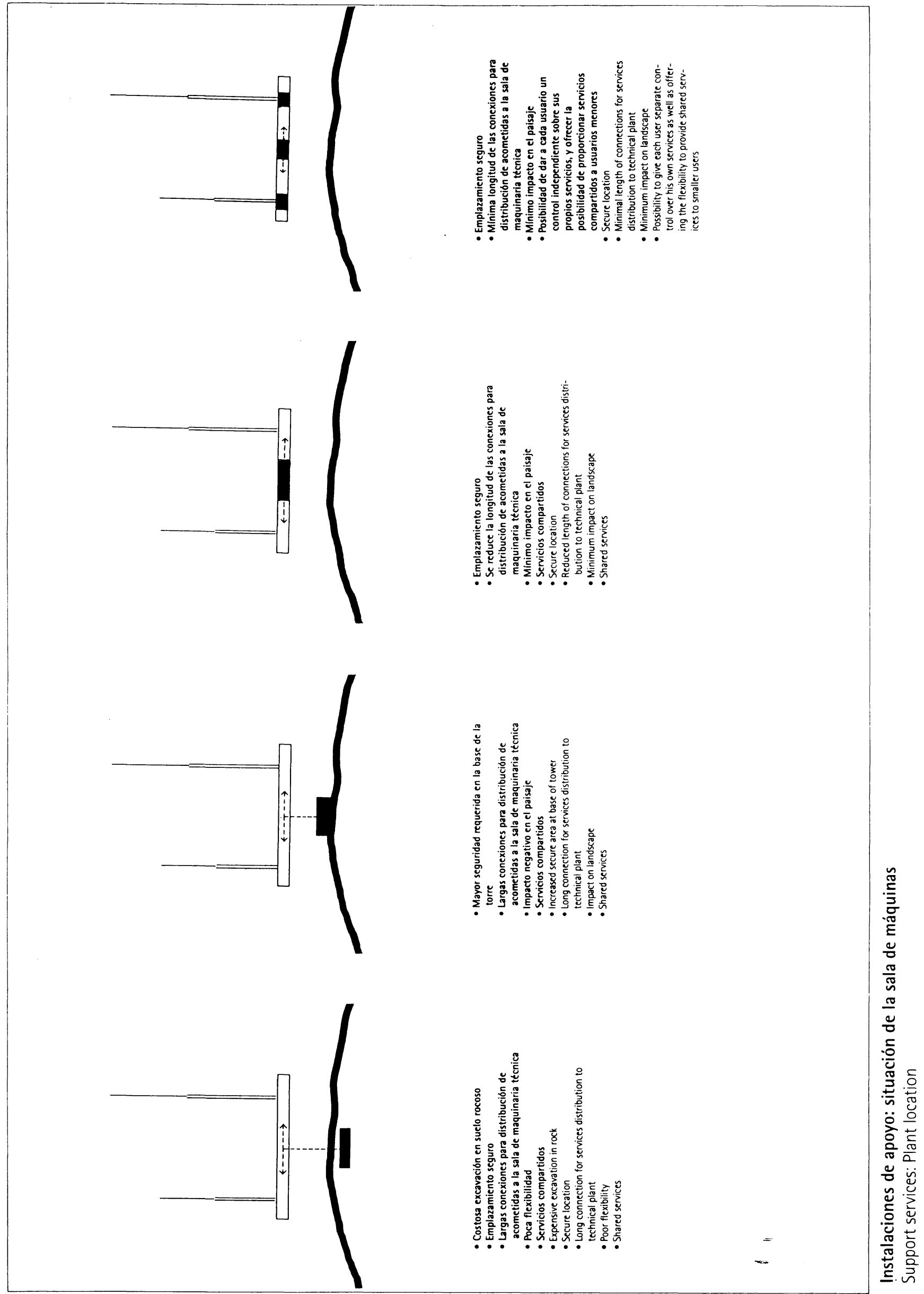




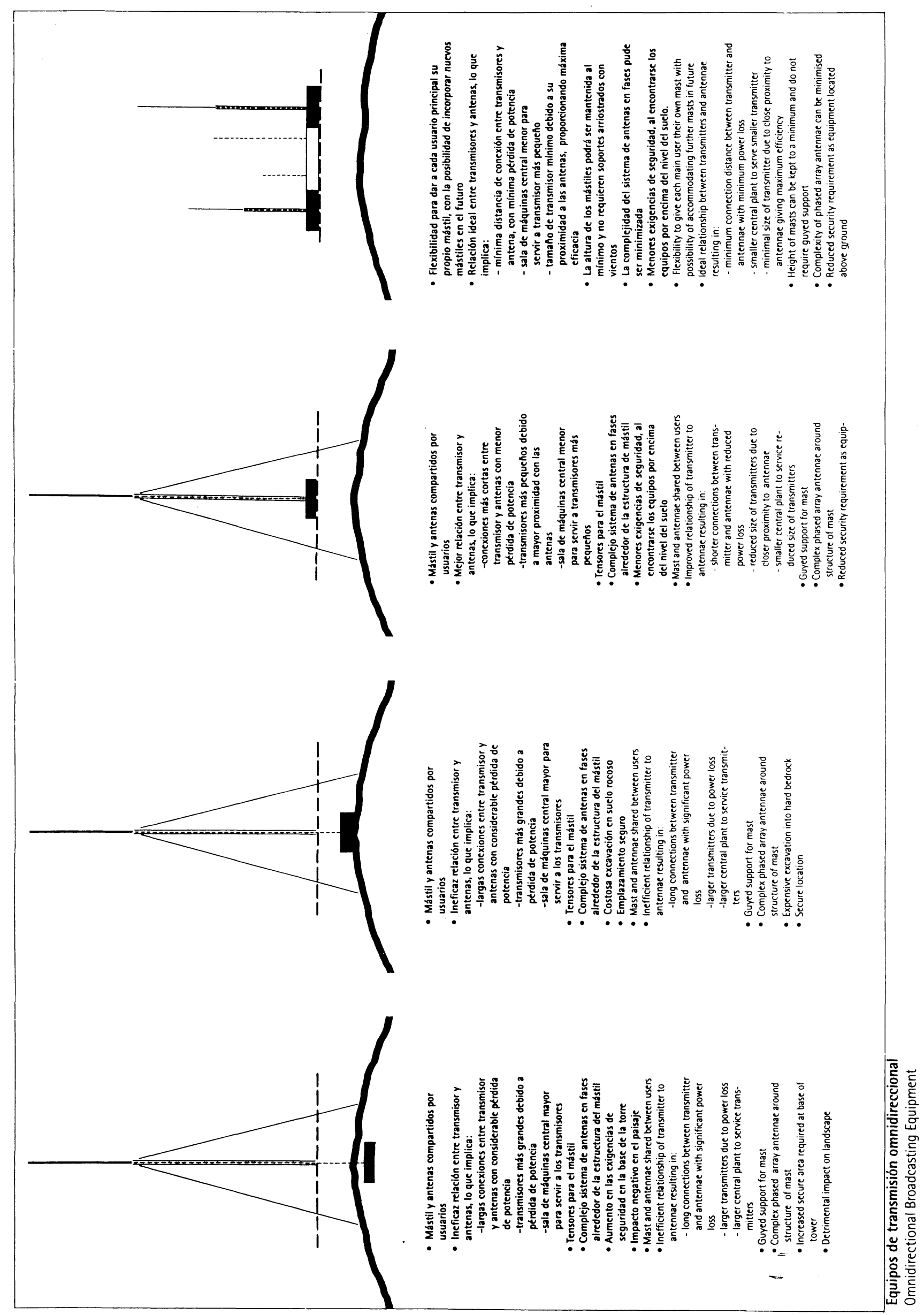



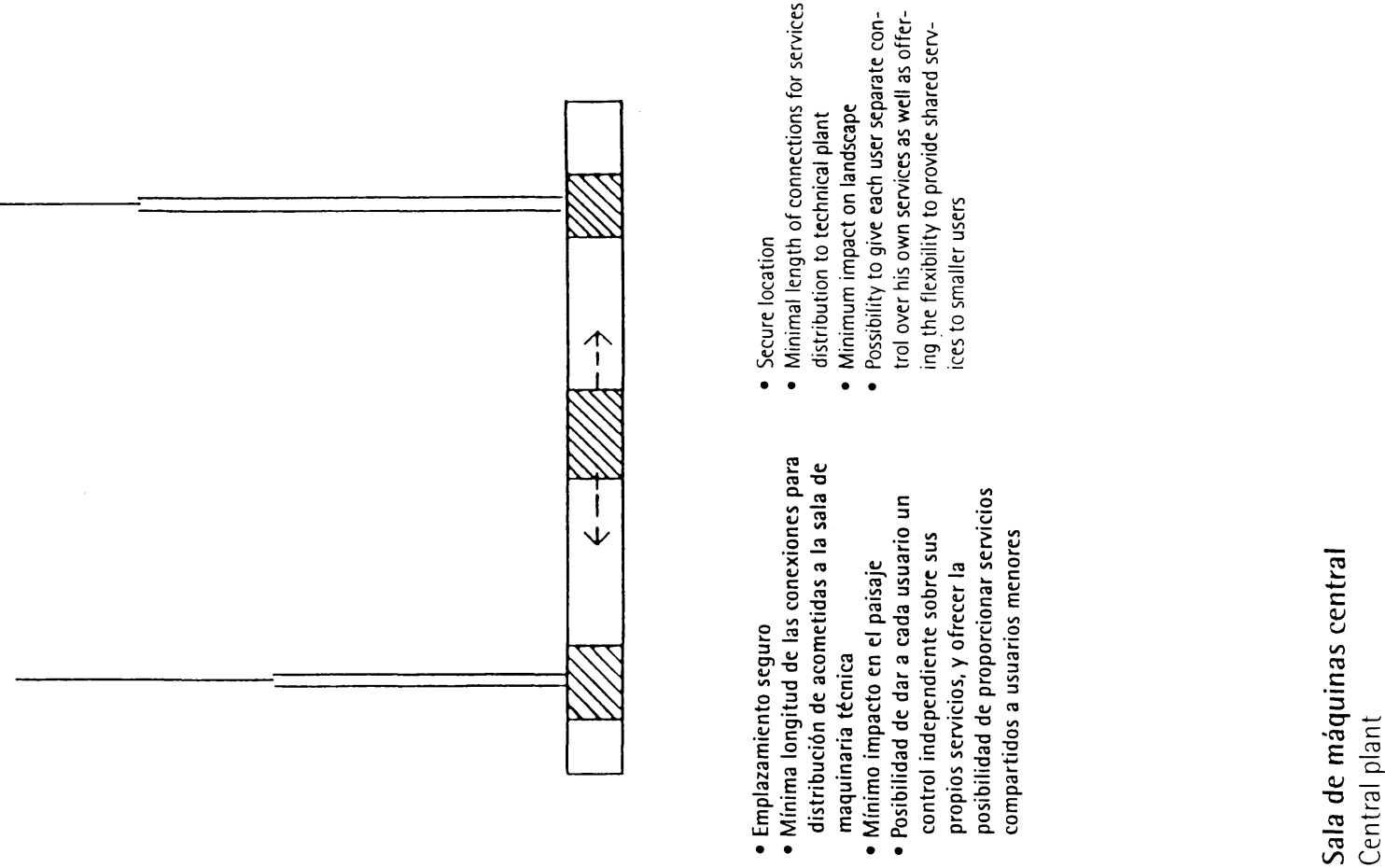


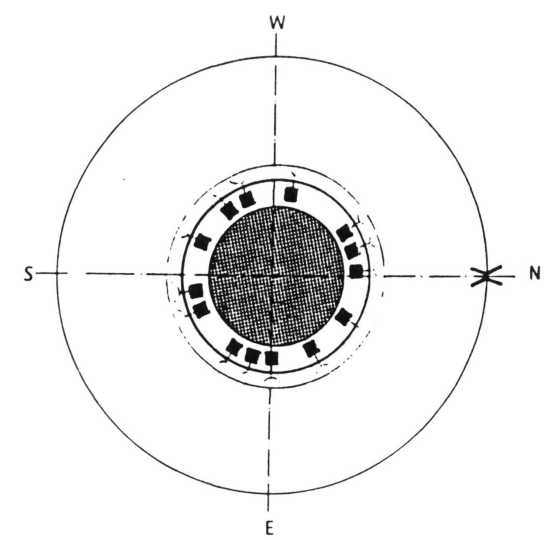

Diagrama de transmisión de microondas ideal:

$-360^{\circ}$ de vistas ininterrumpidas para la antenas

- gran proximidad del transmisor a las

- gran proximic
antenas

antenas proximad de la sala de máquinas

central a los transmisores

- el espacio no tiene suficiente

profundidad en planta para la sala de

máquinas central; requiere una

ubicación alternativa

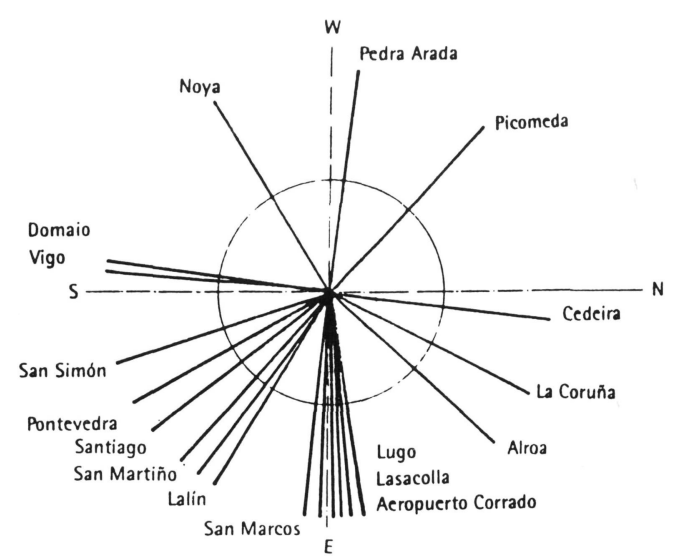

Ideal microwave broadcasting diagram $-360^{\circ}$ unobstructed views for antennae close proximity of transmitter to antennae cose proximity of central plant tc transmil-
ters insufficient deep plan space for centra plant, requires alternative location
Recorridos de microondas actuales demanda de espacio como resultad - non uniform distribution of demand for una orientación asimétrica de los space as a result of asymmetrical orientarecorridos

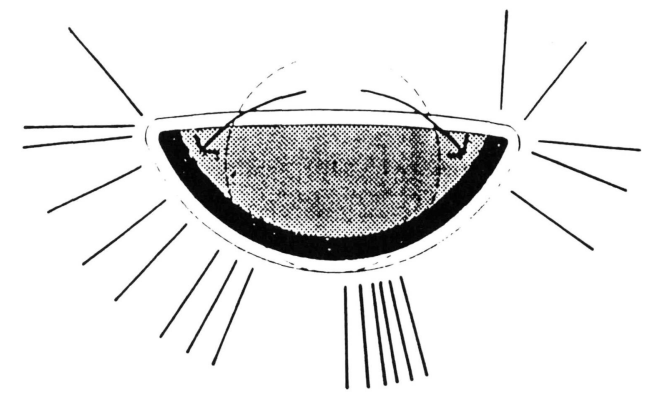

Optimizar la configuración del espacio para satisfacer las necesidades particulares de Santiago Compostela. proporcionando mayor perimetro a las antenas y con arreglo a la demanda de rutas Se proporcion a su vez mayor profundidad en el espacio de planta designado para la sala de máquinas.

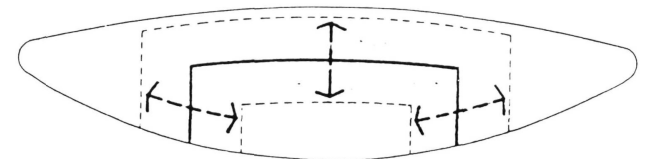

Con una plataforma principal y un entresuelo, el espacio puede ser subdividido en un sector público y un sector privado. proporcionando flexibilidad para incorpora futuros cambios. Ello contribuye a asegura que se haga un uso óptimo de la plataforma

Optimise the configuration of space 10 sult the particular requirements of Santiago de Compostela, providing more perımeter for antennac in accordance with the demand tor routes. This also improves the area of deep plan space for plant
With one main platform and a mezzanine the space can be subdivided between public and private in a way that allows flexibility to accom. modate future change. This helps to ensure that optimum use is made of the platform at all mes.

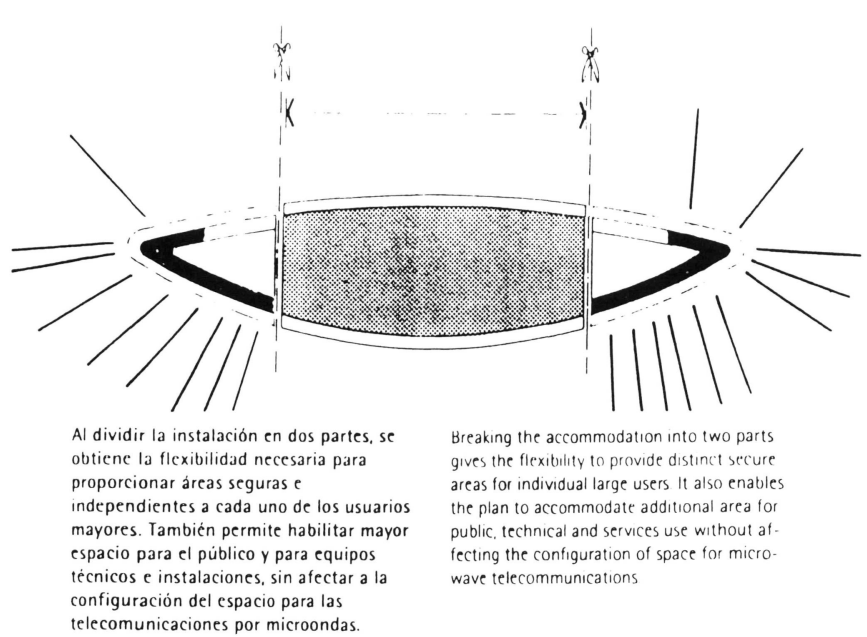

(c) Consejo Superior de Investigaciones Científicas 


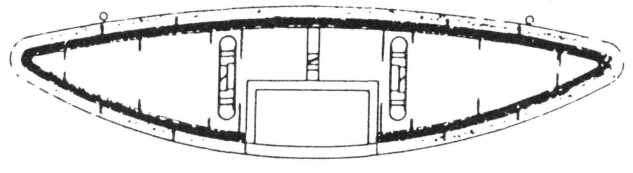

Circulación técnica cerrada al público: planta principal Secure Technical Circulation: Main level

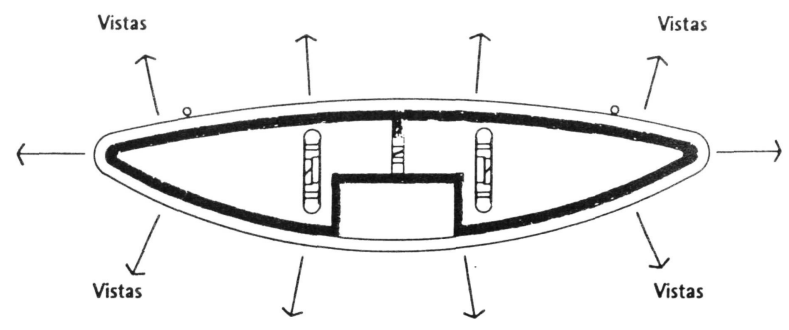

Circulación pública: planta de entresuelo Public Circulation : Mezzanine level

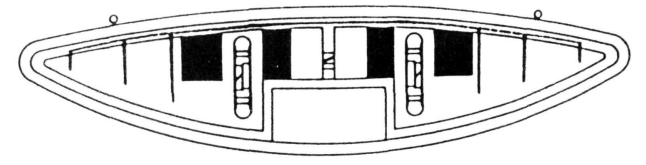

Distribución de las instalaciones principales Main Services Distribution
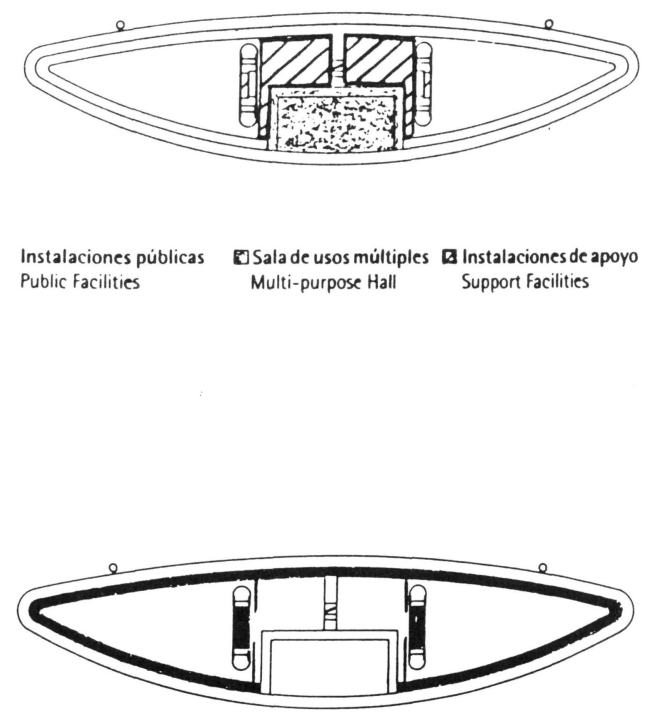

Medios de evacuación: planta principal y planta de entresuelo Means of Escape: Main level and Mezzanine level

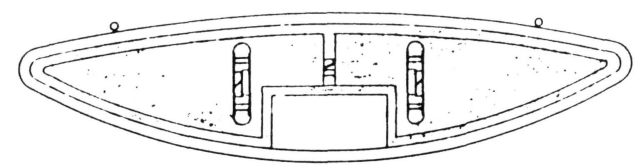

Flexibilidad/expansión: máximo perimetro para el entresuelo Flexibility/Expansion: Maximum extent of Mezzanine

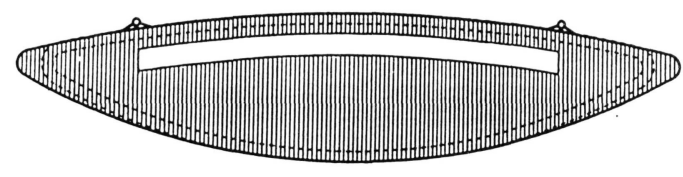

Granja de antenas en la cubierta Roof Antennae Farm
Galeria para antenas: planta principal

Antennae Gallery: Main level

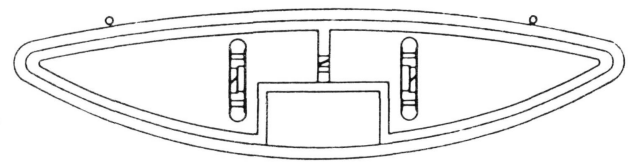



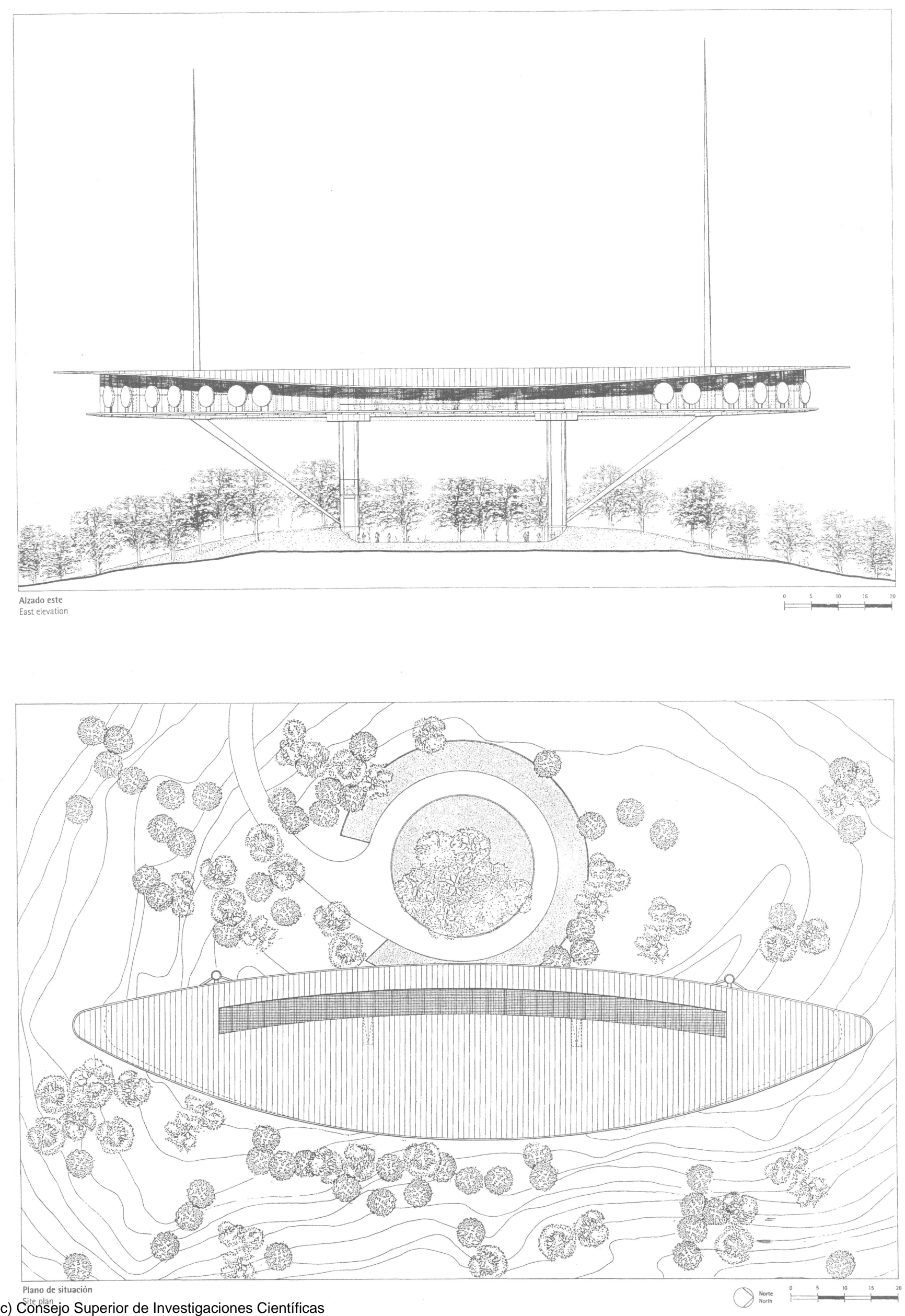

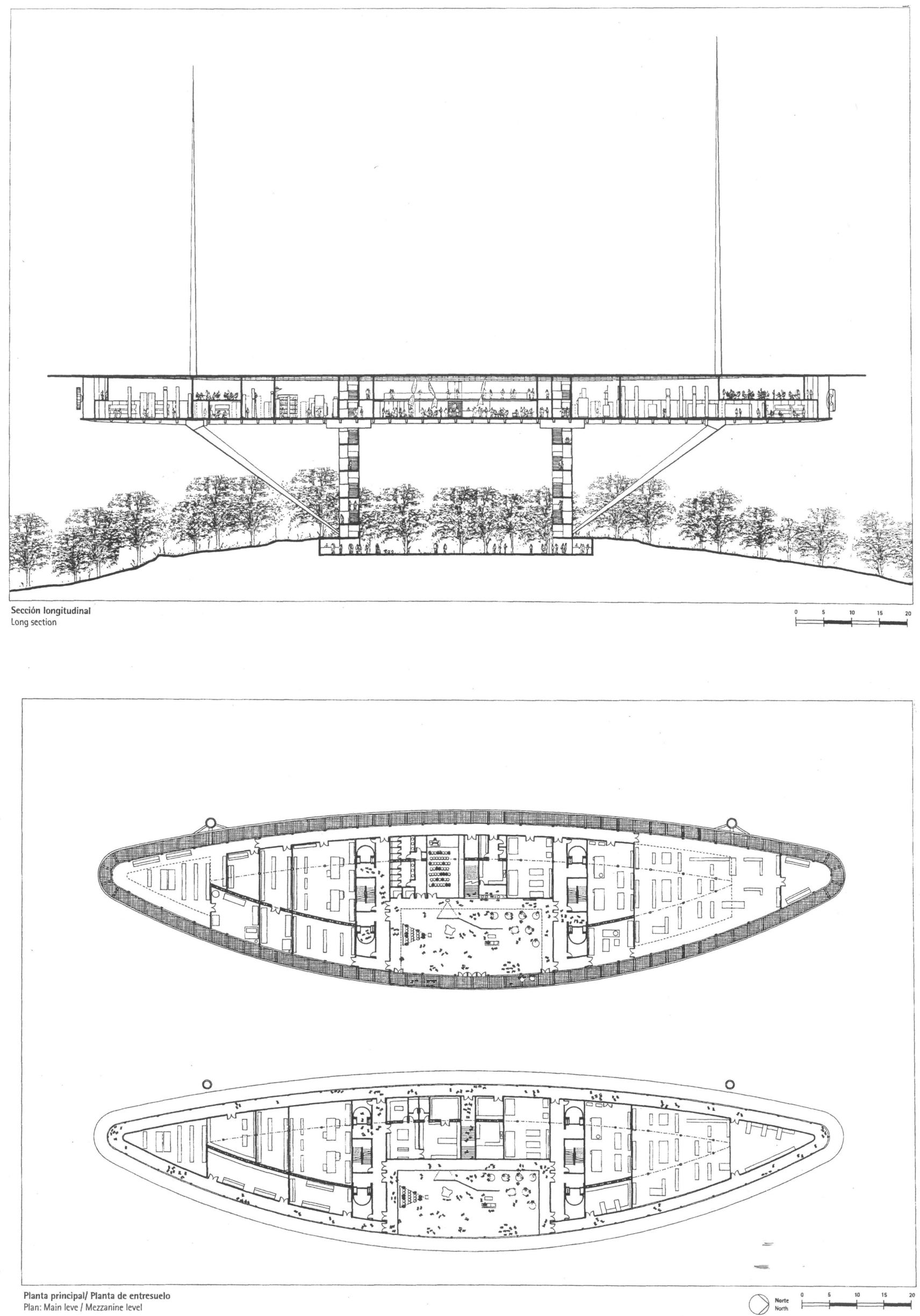

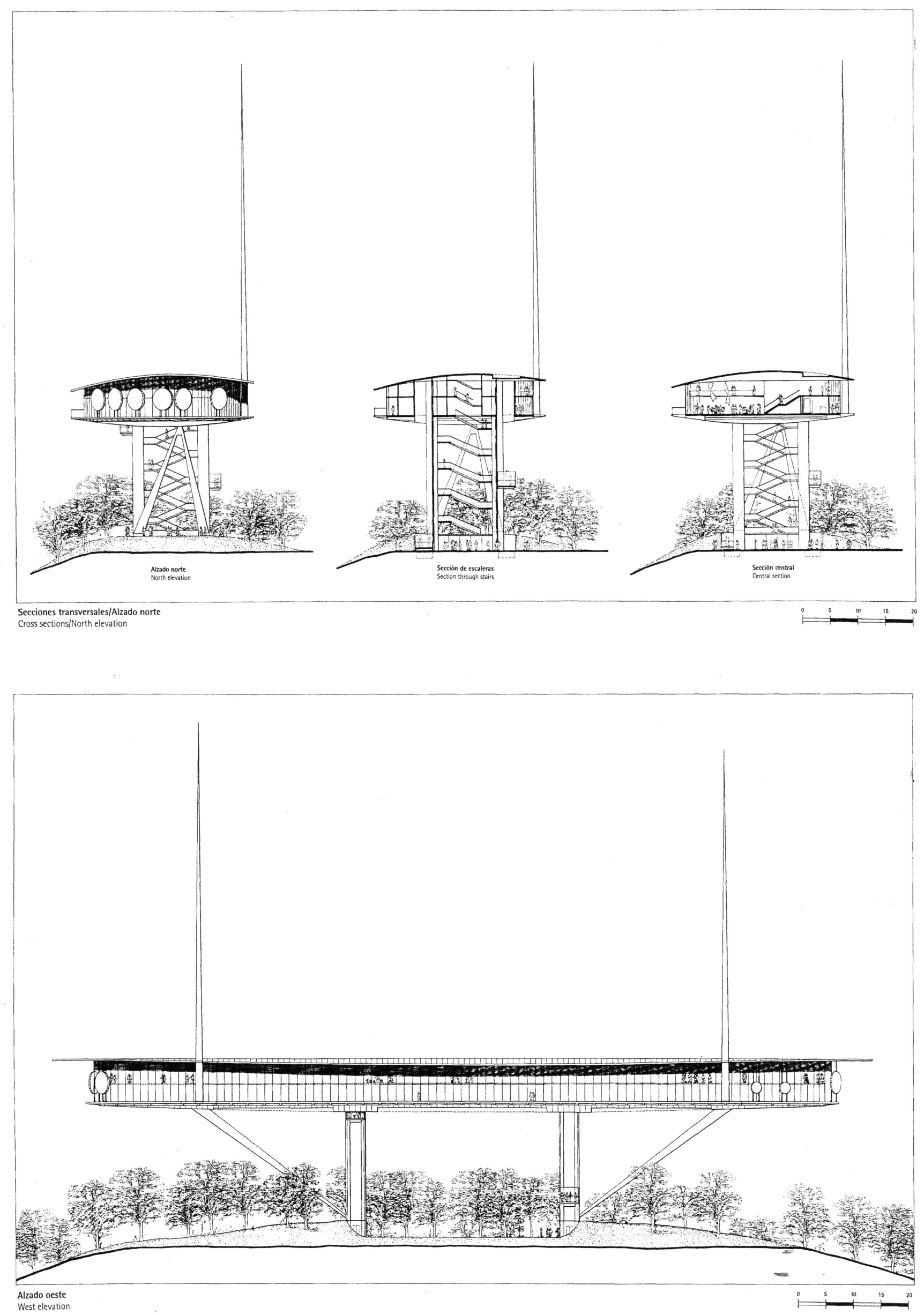

(c) Consejo Superior de Investigaciones Científicas 

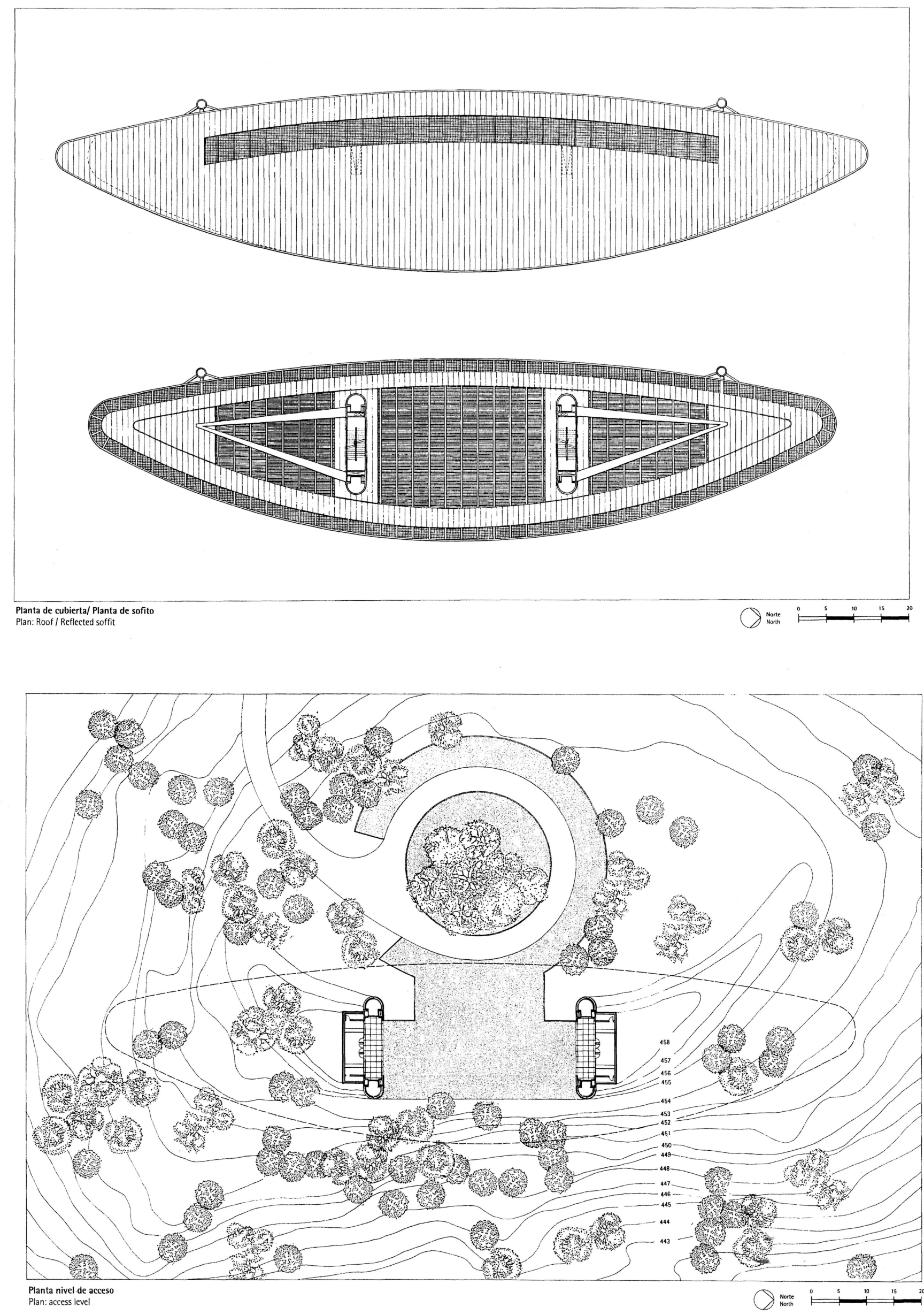